Acetylcholine 198

Acoustic communication 43,70

Active space 83

Allomelry 157

Amphibian visual system 198

Amplitude modulation 134

Androgen 22

Animal communication 32

Anurans 70, 134

Audition 83,95, 145

Auditory system 109, 134

Barn owls 109, 122

Bals 109

Bioacoustics 32

Bird song 22

Body surface area 157

Brain weight 157

Calling 60

Central nervous system 134

Cholinergic drugs 198

CNS divisions 157

Communication of animals 83

'Drum' muscles 7

Echolocation 109

Electric communicaiion 32, 43 
Electric fish 43,60, 122, 134

- organ 7

Electromotoneurons 7

Electroreception 83, 122, 145

Eleclrosensory system 134

Entrainment 60

Enviromental influences 32

Estrogen 22

Features detection 145 


\section{Frogs 60}

Gymnotid fish 170 Hearing 122

Isthmotectal projections 170 Jamming 60 Localization 95 Maps 145

Microiontophoresis 198 Motor nucleus 7 Nucleus isthmi 170 Optic tectum 198

- -, lectoisthmic projections 170 Optokinetic afternystagmus 186

- nystagmus 186 Periodicity pitch 134 Phylogcnetic constraints 70 Recognition 145

Sex Dimorphism 83 Sexual dimorphism 22 Somatic scaling 157 Sonic 'drum' Muscles 7

- $\quad$ motor nucleus 7 Sound localization 109

- transmission 43 Species recognition 43 Spectral cue 95 Temporal 134

- coding 43

- processing 109 Time coding 122 Tonotopy 95

Visual neurophysiology 198 Vocalizations 70 